\title{
AGN feedback in action: a new powerful wind in 1SXPS J050819.8+172149?^
}

\author{
L. Ballo ${ }^{1}$, P. Severgnini ${ }^{1}$, V. Braito ${ }^{2,3}$, S. Campana ${ }^{2}$, R. Della Ceca ${ }^{1}$, A. Moretti ${ }^{1}$, and C. Vignali ${ }^{4,5}$ \\ 1 Osservatorio Astronomico di Brera (INAF), via Brera 28, 20121 Milano, Italy \\ e-mail: lucia.ballo@brera.inaf.it \\ 2 Osservatorio Astronomico di Brera (INAF), via E. Bianchi 46, 23807 Merate, LC, Italy \\ 3 Department of Physics, University of Maryland, Baltimore County, Baltimore, MD 21250, USA \\ 4 Dipartimento di Fisica e Astronomia, Università degli Studi di Bologna, viale Berti Pichat 6/2, 40127 Bologna, Italy \\ 5 Osservatorio Astronomico di Bologna (INAF), via Ranzani 1, 40127 Bologna, Italy
}

Received 21 May 2015 / Accepted 29 June 2015

\section{ABSTRACT}

\begin{abstract}
Context. Galaxy merging is widely accepted to be a driving factor in galaxy formation and evolution, while the feedback from actively accreting nuclei is thought to regulate the black hole-bulge coevolution and the star formation process.

Aims. In this context, we focused on 1SXPS J050819.8+172149, a local $(z=0.0175)$ Seyfert 1.9 galaxy $\left(L_{\text {bol }} \sim 4 \times 10^{43} \mathrm{erg} \mathrm{s}^{-1}\right)$. The source belongs to an infrared-luminous interacting pair of galaxies, characterized by a luminosity for the whole system (due to the combination of star formation and accretion) of $\log \left(L_{\mathrm{IR}} / L_{\odot}\right)=11.2$. We present here the first detailed description of the $0.3-10 \mathrm{keV}$ spectrum of 1SXPS J050819.8+172149, monitored by Swift with nine pointings performed in less than one month.

Methods. The X-ray emission of 1SXPS J050819.8+172149 is analysed by combining all the Swift pointings, for a total of $\sim 72 \mathrm{ks}$ XRT net exposure. The averaged Swift-BAT spectrum from the 70-month survey is also analysed.

Results. The slope of the continuum is $\Gamma \sim 1.8$, with an intrinsic column density of $\sim 2.4 \times 10^{22} \mathrm{~cm}^{-2}$, and a de-absorbed luminosity of $\sim 4 \times 10^{42} \mathrm{erg} \mathrm{s}^{-1}$ in the $2-10 \mathrm{keV}$ band. Our observations provide a tentative $(2.1 \sigma)$ detection of a blueshifted Fe XXVI absorption line (rest-frame $E \sim 7.8 \mathrm{keV}$ ), thus suggesting the discovery of a new candidate powerful wind in 1SXPS J050819.8+172149. The physical properties of the outflow cannot be firmly assessed owing to the low statistics of the spectrum and to the observed energy of the line, too close to the higher boundary of the Swift-XRT bandpass. However, our analysis suggests that, if the detection is confirmed, the line could be associated with a high-velocity $\left(v_{\text {out }} \sim 0.1 c\right)$ outflow most likely launched within $80 r_{\mathrm{S}}$. To our knowledge this is the first detection of a previously unknown ultrafast wind with Swift. The high column density suggested by the observed equivalent width of the line $(E W \sim-230 \mathrm{eV}$, although with large uncertainties) would imply a kinetic output strong enough to be comparable to the AGN bolometric luminosity.
\end{abstract}

Key words. galaxies: active - X-rays: individuals: 1SXPS J050819.8+172149 - quasars: absorption lines - galaxies: star formation

\section{Introduction}

The observational evidence for the presence of inactive super massive black holes (SMBHs; $M_{\mathrm{BH}} \sim 10^{6}-10^{10} M_{\odot}$ ) at the centre of most, if not all, the local galaxies, and the observed correlation between several properties of the galaxy's bulge and the central SMBH mass (Ferrarese \& Merritt 2000; Gebhardt et al. 2000), suggest that the SMBH accretion and the assembly of the galaxies bulges are intimately related (see Kormendy \& Ho 2013 , for a recent review). Funnelling of gas in the nuclear regions, as triggered by galaxy interactions, can activate both efficient accretion onto the SMBH, and a burst of star formation. A key ingredient in regulating their evolution should be the feedback from the active galactic nuclei (AGN); being conservative, while building its mass the SMBH can release an amount of energy larger than $\sim 30$ times the binding energy of the host bulge (see the review presented in Fabian 2012). Even if only a small fraction of this energy is transferred to the gas in the galaxy, an active nucleus can have a profound effect on the evolution of its host (Di Matteo et al. 2005). Powerful (kinetical or radiative) outflows of gas driven by luminous quasars are invoked as one of the main mechanisms that blow away the gas in the galaxy

* Based on observations obtained with the Swift satellite. and thereby quenche star formation, coincidentally starving the SMBH of fuel (King \& Pounds 2015).

Active galactic nucleus winds with a range of physical properties have been revealed by observations at various energies, from radio up to $\mathrm{X}$-rays. Outflows of molecular or neutral atomic gas, with velocities up to $\sim 1000-2000 \mathrm{~km} \mathrm{~s}^{-1}$ and extending on $\mathrm{kpc}$ scales, have been observed at $\mathrm{mm}$ (e.g. Feruglio et al. 2010; Cicone et al. 2014) and radio frequencies (e.g. Morganti et al. 2005; Teng et al. 2013) in a few dozen AGNs in dusty star forming sources and/or radio galaxies. Mass outflows of ionised gas with similar velocities at distances consistent with the narrow line region zone have been detected in the optical/ultraviolet (UV), both in the [O III] emission line profiles (e.g. Crenshaw \& Kraemer 2005; Cano-Díaz et al. 2012; Cresci et al. 2015) and through the observation of broad absorption line systems (e.g. Dai et al. 2008; Borguet et al. 2013). In X-rays, mildly ionised warm absorbers are observed in more than half of unobscured AGNs (Crenshaw et al. 2003; Blustin et al. 2005). The observed velocities of $\sim 500-1000 \mathrm{~km} \mathrm{~s}^{-1}$ imply a kinetic power that is rather low when compared to the bolometric luminosity. However, Crenshaw \& Kraemer (2012) found that, when summed over all the absorbers, the total power carried by these structures can, in some cases, reach the minimum level required 
for AGN feedback $\left(L / L_{\text {bol }} \sim 0.5-5 \%\right.$; e.g. Di Matteo et al. 2005; Hopkins \& Elvis 2010). In recent years, highly blueshifted Fe K-shell absorption lines at rest-frame energies $E \gtrsim 7 \mathrm{keV}$, observed in XMM-Newton or Suzaku spectra of luminous AGN, revealed the presence of high column density $\left(N_{\mathrm{H}} \sim 10^{23} \mathrm{~cm}^{-2}\right)$ and fast $(v>0.1 c)$ winds (e.g. Tombesi et al. 2010; Gofford et al. 2013; Tombesi et al. 2015). Their derived kinetic power is systematically higher than the minimum fraction of bolometric luminosity required by AGN feedback models in order to regulate the growth of an SMBH and its galactic bulge (e.g. Hopkins \& Elvis 2010). Only very recently, outflows over a range of scales have been detected and studied within the same source, thus allowing us to explore the connection between large-scale molecular outflows and accretion-disk activity (Feruglio et al. 2015; Tombesi et al. 2015).

Finding observational evidence of the effects of such accretion-related feedback and characterising the magnitude of mass outflows from AGN are among the major challenges of the current extragalactic astronomy. Here we present nine coadded Swift observations (performed in less than one month) of the interacting infrared (IR) galaxy 1SXPS J050819.8+172149. The spectrum, the first for this source covering the energy range $E \sim 7-10 \mathrm{keV}$, provides us with a tentative detection of a possible new ultrafast wind $\left(v_{\text {out }} \sim 0.1 c\right)$. To our knowledge, this is the first time that a previously unknown outflow has been revealed by Swift.

The source is described in Sect. 2, while the analysis of the Swift-XRT data is presented in Sect. 3 and the results are summarised in Sect. 4. Throughout the paper we assume a flat $\Lambda \mathrm{CDM}$ cosmology with $H_{0}=71 \mathrm{~km} \mathrm{~s}^{-1} \mathrm{Mpc}^{-1}, \Omega_{\Lambda}=0.7$, and $\Omega_{\mathrm{M}}=0.3$.

\section{1SXPS J050819.8+172149}

The position of the Swift source 1SXPS J050819.8+172149 (Evans et al. 2014) is coincident with the centre of CGCG 468002 NED01, a local $(z=0.0175)$ galaxy belonging to the interacting pair CGCG 468-002 (projected distance between the centre of the galaxies of $\sim 29.4^{\prime \prime}$, corresponding to $\sim 10 \mathrm{kpc}$ at the source redshift).

The high $8-1000 \mu \mathrm{m}$ luminosity of the system, $\log \left(L_{\mathrm{IR}} / L_{\odot}\right)=11.2$ (Armus et al. 2009), implies a classification as luminous infrared galaxy (LIRG, defined as having $L_{\mathrm{IR}} \simeq 10^{11-12} L_{\odot} ;$ Sanders \& Mirabel 1996). Included for this reason in the Great Observatories All-Sky LIRG Survey (GOALS; Armus et al. 2009), the system has been targeted by several observational facilities, and multiwavelength information (both spectroscopic and photometric) has been collected. The available data span from the UV (GALEX; Howell et al. 2010) up to the mid-infrared (mid-IR; Spitzer/IRS, IRAC, and MIPS; Díaz-Santos et al. 2010, 2011; Petric et al. 2011; Inami et al. 2013; Stierwalt et al. 2013, 2014, see also Valiante et al. 2009; Alonso-Herrero et al. 2012, 2013) and far-infrared (Herschel/PACS; Díaz-Santos et al. 2013) bands. Photometry from the 2MASS Redshift Survey is reported by Huchra et al. (2012). At radio wavelengths, the relatively strong $\left(S_{v} \sim 34 \mathrm{mJy}\right)$ NVSS emission (Condon et al. 1998), detected halfway between the nuclei and slightly elongated in their direction, is probably due to the combined contribution of both galaxies.

The system is an early-stage merger (Stierwalt et al. 2013) known to host an AGN optically classified as Seyfert 1.9 (see e.g. Motch et al. 1998; Véron-Cetty \& Véron 2001; Kollatschny et al. 2008). Mainly based on the detection of the high-ionisation line [Ne V] in the Spitzer/IRS spectra, the Seyfert 1.9 nucleus has been associated with the western galaxy of the system, coincident with 1SXPS J050819.8+172149 (Petric et al. 2011; Alonso-Herrero et al. 2012; Stierwalt et al. 2013, 2014, see also the optical classification reported by Alonso-Herrero et al. 2013). The Spitzer spectra are suggestive of a relatively unobscured AGN (consistent with the optical classification as Seyfert 1.9), energetically important in the mid-IR (Stierwalt et al. 2013). From a decomposition of the mid-IR spectra into AGN and starburst components, Alonso-Herrero et al. (2012) estimated a bolometric luminosity due to the accretion of $L_{\text {bol }} \sim$ $10^{10} L_{\odot}$. Instead, no signature of active accretion is found for the eastern galaxy, which shows all the typical properties of a star forming source both in the mid-IR spectra and from the UV photometry.

The BH mass in 1SXPS J050819.8+172149 is among the highest observed in local LIRGs, $M_{\mathrm{BH}} \sim 1.15 \times 10^{8} M_{\odot}$ (as calculated from the velocity dispersion of the core of the [O III] $\lambda 5007$ line; Alonso-Herrero et al. 2013). This implies that the black hole is radiating at a low fraction of its Eddington luminosity ${ }^{1}$, with Eddington ratio $\log \lambda_{\text {Edd }} \equiv \log L_{\text {bol }} / L_{\text {Edd }} \sim-2.5$. The star formation rate (SFR) derived for this source falls in the lower tail of the distribution found for local LIRGs (nuclear $S F R \sim 1-3 M_{\odot} \mathrm{yr}^{-1}$, integrated $S F R \sim 3-4 M_{\odot} \mathrm{yr}^{-1}$, Alonso-Herrero et al. 2013), implying a ratio between SFR and $\mathrm{BH}$ accretion rate of $\log S F R / \dot{m}_{\mathrm{BH}} \sim 2$ (by assuming a massenergy conversion efficiency $\epsilon=0.1$ ), similar to the values found for Seyfert galaxies.

\section{XRT data analysis}

In addition to the basic analysis reported in the Swift-XRT point source catalogue (Evans et al. 2014), in the soft-medium $\mathrm{X}$-ray energy range $(E \lesssim 10 \mathrm{keV})$ the only published information to date comes from the ROSAT All Sky Survey (see Kollatschny et al. 2008, and references therein), providing a soft X-ray luminosity (de-absorbed by our Galaxy)

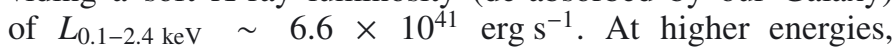
1SXPS J050819.8+172149 has been detected by the Swift-Burst Alert Telescope (BAT; see Baumgartner et al. 2013), while Ackermann et al. (2012) reported only a Fermi $95 \%$ confidence level upper limit of $L_{0.1-10 \mathrm{GeV}} \sim 1.6 \times 10^{42} \mathrm{erg} \mathrm{s}^{-1}$.

Recently, our group has been awarded a Swift (Gehrels et al. 2004) program for this source (PI P. Severgnini): nine pointings performed with the X-ray telescope (XRT; Burrows et al. 2005 ) in the standard photon counting (PC) mode between 2014 October 15 and 2014 November 11, for a total of $\sim 72 \mathrm{ks}$ net exposure (ObsID from 00049706003 to 00049706011 ; see Table 1).

We generated images, light curves, and spectra, including the background and ancillary response files, with the online XRT data product generator ${ }^{2}$ (Evans et al. 2007, 2009); the appropriate spectral response files have been identified in the calibration database. The source appears point-like in the XRT image and centred at the position of the western nucleus, without any evident elongation toward the position of the second galaxy. We note that at the angular resolution of XRT, $18^{\prime \prime}$ half-power diameter, the emission of the two galaxies,

1 The Eddington luminosity, defined as $L_{\mathrm{Edd}}=4 \pi G M_{\mathrm{BH}} m_{\mathrm{p}} c / \sigma_{\mathrm{T}} \simeq$ $1.3 \times 10^{38} M_{\mathrm{BH}} / M_{\odot}\left[\mathrm{erg} \mathrm{s}^{-1}\right.$, represents the exact balance between inward gravitational force and outward radiation force acting on the gas, assumed to be of ionised hydrogen in a spherical configuration.

2 http://www.swift.ac.uk/user_objects 
Table 1. Swift-XRT monitoring observation $\log$ for 1SXPS J050819.8+172149.

\begin{tabular}{lccc}
\hline \hline Obs. ID & Start date & $\begin{array}{c}\text { Net count rate } \\
{\left[10^{-2} \text { counts s }^{-1}\right]}\end{array}$ & $\begin{array}{c}\text { Net exp. time } \\
{[\mathrm{s}]}\end{array}$ \\
\hline 00049706003 & 2014-Oct.-15 & $7.5 \pm 0.4$ & 4235 \\
00049706004 & 2014-Oct.-16 & $13.1 \pm 0.3$ & 14740 \\
00049706005 & 2014-Oct.-21 & $10.0 \pm 0.7$ & 2308 \\
00049706006 & 2014-Oct.-22 & $7.3 \pm 0.3$ & 7325 \\
00049706007 & 2014-Oct.-23 & $7.5 \pm 0.3$ & 6533 \\
00049706008 & 2014-Oct.-27 & $5.8 \pm 0.3$ & 6478 \\
00049706009 & 2014-Oct.-28 & $5.9 \pm 0.3$ & 6218 \\
00049706010 & 2014-Oct.-30 & $4.6 \pm 0.3$ & 6333 \\
00049706011 & 2014-Nov.-11 & $7.6 \pm 0.2$ & 17950 \\
\hline
\end{tabular}

Notes. Net count rates are in the $0.3-10 \mathrm{keV}$ energy range.

located at a distance of $\sim 29.4^{\prime \prime}$, can be resolved; by assuming a power-law model with $\Gamma=2$, we estimated a $3 \sigma$ upper limit to the $0.3-10 \mathrm{keV}$ emission at the eastern source position of $\sim 7 \times 10^{-14} \mathrm{erg} \mathrm{cm}^{-2} \mathrm{~s}^{-1}$.

Source events were extracted from a circular region with a radius of 20 pixels (which corresponds to an encircled energy fraction of 90\%, Moretti et al. 2005, 1 pixel $\sim 2.36$ arcsec), while background events were extracted from an annulus region centred on the source with inner and outer radii of 60 and 180 pixels, respectively; all sources identified in the image were removed from the background region.

We extracted a light curve binned at the duration of each individual observation. 1SXPS J050819.8+172149 was detected in all observations, with $0.3-10 \mathrm{keV}$ signal-to-noise ratios $(\mathrm{S} / \mathrm{N})$ ranging from 15 to 44 . The average count rates in the total $(0.3-10 \mathrm{keV})$, soft $(0.3-2 \mathrm{keV})$, and hard (2-10 keV) XRT energy ranges are $\sim 0.09, \sim 0.03$, and $\sim 0.07$ counts $\mathrm{s}^{-1}$, respectively. Small deviations from these values, of a factor lower than 2, are observed in the light curves; however, there is no evidence of spectral variability in the ratio of count rates observed in the hard and soft bands. We do not find any significant pile-up problem.

In order to increase the statistics, we co-added the XRT datasets. Source and background spectra were extracted from the merged event lists, and the former was binned in order to have at least 20 total counts per energy channel. The net count rates in the $0.3-10 \mathrm{keV}, 0.3-2 \mathrm{keV}$, and $2-10 \mathrm{keV}$ energy ranges are $(8.2 \pm 0.1) \times 10^{-2},(2.3 \pm 0.1) \times 10^{-2}$, and $(5.8 \pm 0.1) \times 10^{-2}$ counts $\mathrm{s}^{-1}$, respectively. The $\mathrm{S} / \mathrm{N}$ achieved in the same energy ranges are 76, 40, and 64, respectively.

Spectral fits were performed in the $0.3-10 \mathrm{keV}$ energy range using the X-ray spectral fitting package XSPEC (Arnaud 1996) v12.8.2. Uncertainties are quoted at the $90 \%$ confidence level for one parameter of interest $\left(\Delta \chi^{2}=2.71\right)$. All the models discussed in the following assume Galactic absorption with a column density of $N_{\mathrm{H}, \mathrm{Gal}}=1.84 \times 10^{21} \mathrm{~cm}^{-2}$ (Kalberla et al. 2005). To model both Galactic and intrinsic absorptions we used the ( $\mathrm{z}$ )PHABS model in XSPEC, adopting cross-sections and abundances of Wilms et al. (2000).

A fit with a simple absorbed power law clearly provides a poor representation of the XRT data, $\chi^{2}=280.8$ for 229 degrees of freedom (d.o.f.); the photon index is $\Gamma=1.49 \pm 0.09$ and the column density is $N_{\mathrm{H}}=(1.8 \pm 0.2) \times 10^{22} \mathrm{~cm}^{-2}$. Above $\sim 5 \mathrm{keV}$, i.e. in the energy range where the iron $\mathrm{K}$ complex is expected, residuals are present both in emission and in absorption (see Fig. 1), while a big bump below $1 \mathrm{keV}$ suggests that an additional component is emerging in the soft band. Assuming that the neutral absorber only partially covers the central source

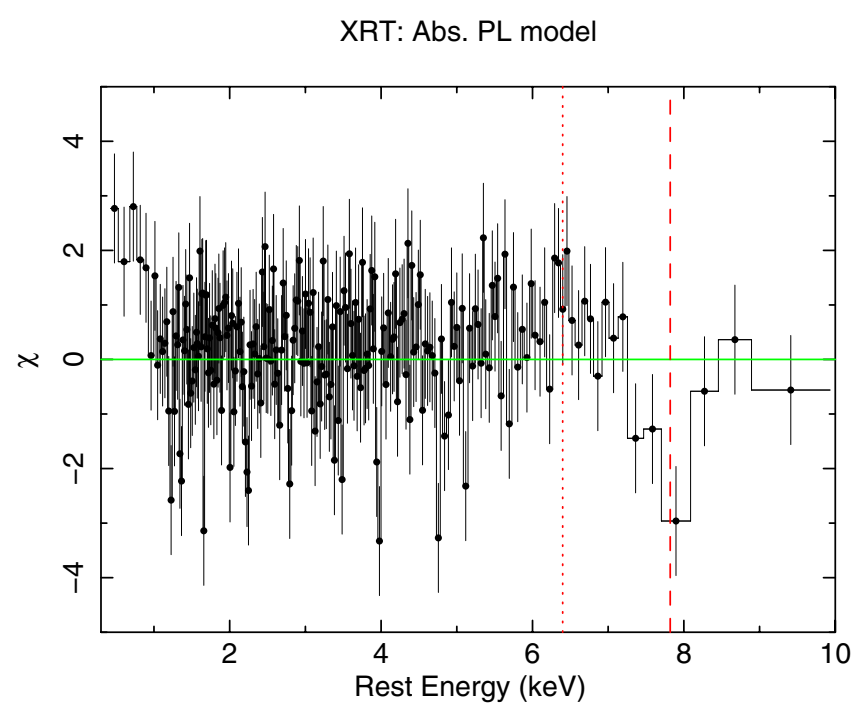

Fig. 1. Residuals, plotted in terms of sigma with error bars of size one, for the XRT spectrum, modelled with an absorbed power-law component $\left(\Gamma \sim 1.5, N_{\mathrm{H}} \sim 1.8 \times 10^{22} \mathrm{~cm}^{-2}\right)$. A deep absorption trough is seen in the iron $K$ band, possibly associated with a broad absorption feature (dashed red line). The dotted red line marks the position where the neutral $\mathrm{Fe} \mathrm{K} \alpha$ emission line is expected (and marginally detected).

(ZPCFABS model in XSPEC) still leaves residuals in the soft band $\left(\chi^{2} /\right.$ d.o.f. $\left.=271.0 / 228\right)$; we found a covering fraction $\gtrsim 0.95$, with $\Gamma=1.48 \pm 0.04$ and $N_{\mathrm{H}}=(2.1 \pm 0.1) \times 10^{22} \mathrm{~cm}^{-2}$.

In principle, the emission observed at low energies can be associated with the accreting nucleus (i.e. due to optically ionised thin gas scattering off the intrinsic radiation) and/or the host galaxy (a soft thermal emission is a characteristic signature in all known starburst galaxies). Phenomenologically, the observed soft excess can be accounted for equally well by additions to the previous model: 1) an unabsorbed power law with photon index $\Gamma$ tied to that of the primary power law $\left(\chi^{2} /\right.$ d.o.f. $\left.=258.4 / 228\right)$; the best-fit parameters are $\Gamma=1.57 \pm$ $0.09, N_{\mathrm{H}}=(2.2 \pm 0.2) \times 10^{22} \mathrm{~cm}^{-2}$, and ratio of the powerlaw normalisations $A_{\text {scatt }} / A_{\text {intr }} \sim 3 \%$; or 2 ) a thermal component (MEKAL model in XSPEC; $\chi^{2} /$ d.o.f. $=259.3 / 227$ ); in this case we found $\Gamma=1.53 \pm 0.09, N_{\mathrm{H}}=(1.9 \pm 0.2) \times 10^{22} \mathrm{~cm}^{-2}$, and $k T=0.31_{-0.09}^{+0.24} \mathrm{keV}$. In the second parametrisation, the luminosity attributed to the thermal component is $L_{0.5-2 \mathrm{keV}}^{\mathrm{mek}} \sim$ $2.6 \times 10^{40} \mathrm{erg} \mathrm{s}^{-1}$, which would imply a $S F R \sim 0.5-2.9 M_{\odot} \mathrm{yr}^{-1}$ (e.g. Ranalli et al. 2003; Mas-Hesse et al. 2008; Mineo et al. 2012), consistent with the nuclear star formation properties of the host (see Sect. 2). In both cases, the luminosity observed in the range covered by ROSAT, $L_{0.1-2.4 \mathrm{keV}} \sim 5.6 \times 10^{41} \mathrm{erg} \mathrm{s}^{-1}$, is in agreement with the value derived by Kollatschny et al. (2008).

While the most plausible hypothesis is that both components contribute to the emission observed at low energies in the XRT spectrum, the quality of the present data does not allow us to discriminate between the two contributions, and even less to disentangle them. In the following, we assume an unabsorbed power law, checking that the inclusion of a thermal component in place of the power law does not affect the main results presented here.

The addition of a narrow (50 eV) Gaussian emission line to the absorbed plus unabsorbed power laws results in an improvement in the fit $\left(\Delta \chi^{2} / \Delta\right.$ d.o.f. $\left.=11.3 / 2\right)$. The line parameters are $E=6.40 \pm 0.09 \mathrm{keV}$, consistent with neutral $\mathrm{Fe} \mathrm{K} \alpha$, $I=1.5 \pm 0.7 \times 10^{-5}$ photons $\mathrm{cm}^{-2} \mathrm{~s}^{-1}$, and $E W \sim 200 \mathrm{eV}$. 
The strength of the $\mathrm{Fe} \mathrm{K} \alpha$ line, coupled with the hard photon index $(\Gamma \sim 1.62)$, could suggest the presence of neutral reflection (Reynolds et al. 1994; Matt et al. 1996, 2000). This possible component was then included in the model by replacing the narrow Gaussian line with a PEXMON component (Nandra et al. 2007), an additive model self-consistently incorporating the Compton-reflected continuum from a neutral slab combined with emission from $\mathrm{Fe} \mathrm{K} \alpha, \mathrm{Fe} \mathrm{K} \beta$, Ni $\mathrm{K} \alpha$, and the Fe K $\alpha$ Compton shoulder.

To improve the determination of the slope of the primary power law and the amount of reflection, it is fundamental to know the shape and intensity of the emission at energies higher than $10 \mathrm{keV}$. Therefore, we fitted the XRT data simultaneously with the averaged Swift-BAT spectrum of 1SXPS J050819.8+172149 obtained from the 70-month survey archive $^{3}$ (SWIFT J0508.1+1727). The data reduction and extraction procedure of the eight-channel spectrum is described in Baumgartner et al. (2013). To fit the pre-processed, backgroundsubtracted BAT spectrum, we used the latest calibration response as of 2013 May: 1SXPS J050819.8+172149 was detected in the 14-100 keV band with a count rate of $(3.9 \pm$ $0.3) \times 10^{-5}$ counts s$^{-1}$, which corresponds to a $14-195 \mathrm{keV}$ flux of $(2.6 \pm 0.5) \times 10^{-11} \mathrm{erg} \mathrm{cm}^{-2} \mathrm{~s}^{-1}$ (Baumgartner et al. 2013).

During the fit, the only free parameter of the PEXMON component was the reflection scaling factor, $R$. We tied the PEXMON photon index and normalization to that of the primary power law, and we fixed the cutoff energy at $1000 \mathrm{keV}$ (i.e. consistent with no measurable cut-off), the inclination angle at $60^{\circ}$, and the abundances of heavy elements at their solar values. We also allow the cross-normalisation factor between the XRT data and the average BAT spectrum to vary.

The baseline model then consists of an absorbed power law and an unabsorbed one plus a reflection component: the photon index and intrinsic absorption of the primary emission are $\Gamma=1.75 \pm 0.09$ (in agreement with the values typically observed in unobscured AGNs; Piconcelli et al. 2005; Mateos et al. 2010; Corral et al. 2011) and $N_{\mathrm{H}}=(2.4 \pm 0.2) \times 10^{22} \mathrm{~cm}^{-2}$, while the reflection fraction and the strength of the scattered component are $R \sim 1.1$ and $A_{\text {scatt }} / A_{\text {intr }} \sim 2 \%$. The cross-normalisation factor between the XRT data and the average BAT spectrum is 0.96 .

Although this model provides an acceptable description of the broad-band $(0.3-100 \mathrm{keV}) \mathrm{X}$-ray continuum of 1SXPS J050819.8+172149 $\left(\chi^{2} /\right.$ d.o.f. $\left.=250.2 / 231\right)$, it is not able to account for the residuals observed above $7 \mathrm{keV}$, confirming the presence of a deep absorption trough at about $8 \mathrm{keV}$ (see Fig. 2). Considering only the 41 XRT bins between 5 and $9 \mathrm{keV}$, the fit statistics provided by the model is $\chi^{2} /$ d.o.f. $=44.2 / 36$. These residuals can be accounted for by adding a Gaussian absorption line, with a rest-frame energy of $E=7.8 \pm 0.3 \mathrm{keV}$ and an equivalent width $E W=-\left(230_{-170}^{+390}\right) \mathrm{eV}$ $\left(\Delta \chi^{2} / \Delta\right.$ d.o.f. $=8.3 / 3$ in the $5-9 \mathrm{keV}$ interval $)$. The line is marginally resolved; allowing its width to vary, we can set only an upper limit $\sigma_{\mathrm{E}}<270 \mathrm{eV}$.

The best-fitting parameters are reported in Table 2, while the unfolded XRT and BAT spectra are shown in Fig. 3. We note that the significance of the detection can depend on the accuracy of the determination of the continuum shape. In particular, we checked if the observed shape can be explained by a mix of a stronger reflection edge plus a steeper and lower continuum. However, even if we assume the combination of reflection strength and intrinsic spectral shape that minimises the intensity

\footnotetext{
3 http://swift.gsfc.nasa.gov/results/bs70mon/SWIFT_ J0508.1p 1727
}

Baseline model: Abs. + unabs. PLs + reflected cont.

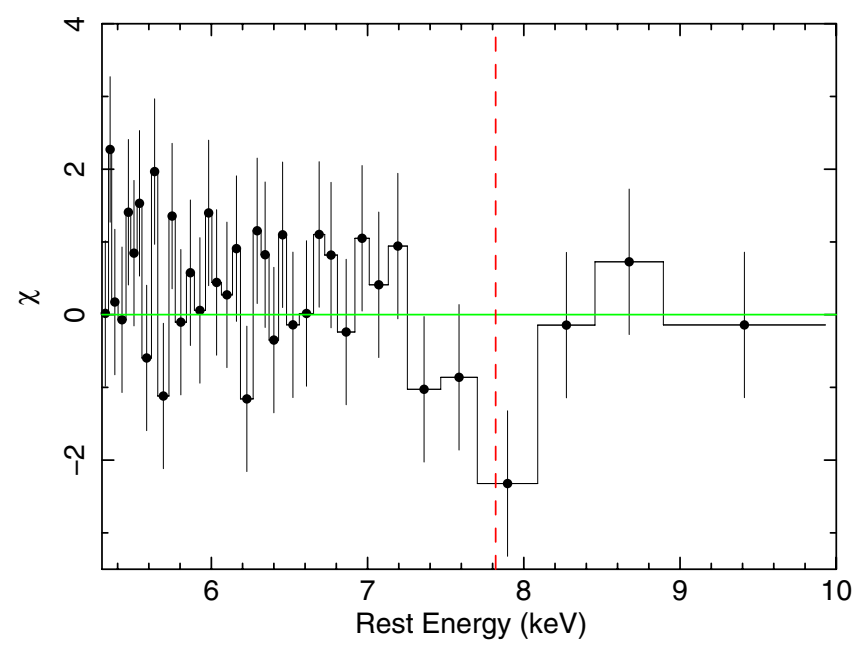

Fig. 2. Residuals, plotted in terms of sigma with error bars of size one, for the XRT spectrum above $5.3 \mathrm{keV}$. The adopted model is an absorbed power law and a reflection component $\left(\Gamma \sim 1.8, N_{\mathrm{H}} \sim 2.4 \times 10^{22} \mathrm{~cm}^{-2}\right.$, $R \sim 1.1)$. An unabsorbed power law with $\sim 2 \%$ of the intensity of the intrinsic power law accounts for the emission below $\sim 1 \mathrm{keV}$ (actually, below the reported energy interval). The centroid of the absorption line when one more Gaussian is included in the model is marked with a red vertical line (absorption line at $E \sim 7.8 \mathrm{keV}$ ).

of the trough, the normalisation of the Gaussian line is still inconsistent with the null value at a confidence level of $90 \%$, in agreement with the significance of the detection derived from the simulations (see below).

The high energy range where the line is observed, very near to the end of the XRT bandpass, could raise concerns of possible artefacts due to the background. However, the $\mathrm{S} / \mathrm{N}$ and number of source counts collected between 8 and $10 \mathrm{keV}(\sim 7$ and $\sim 56$ counts, respectively; last three bins in Fig. 2) strongly support that the spectral rising that defines the line is real. We performed extensive simulations testing the null hypothesis that the spectrum is well fitted by a model that does not include the $7.8 \mathrm{keV}$ absorption feature, as done in Markowitz et al. (2006, see also Porquet et al. 2004). Briefly, to take the observed background and the uncertainty in the continuum into account, we first generated a fake spectrum for a $72 \mathrm{ks}$ exposure assuming the best fit found for the continuum. We then fitted this model to the fake spectrum, and starting from the new best-fit parameters we re-ran a simulation with the same exposure. The baseline model was fitted to this final fake spectrum, and the derived $\chi^{2}$ was compared with the minimum value of $\chi^{2}$ obtained when a narrow $(\sigma=10 \mathrm{eV})$ Gaussian component was included. We stepped the centroid energy of the absorption line over the 6.5-9 keV range in increments of $0.125 \mathrm{keV}$, fitting separately each time to derive the lowest value of $\chi^{2}$. The whole process was repeated 400 times, and we estimated a $4 \%$ probability of detecting a similar feature by chance.

The observed energy of the line suggested by the data is not consistent with any of the atomic transitions ${ }^{4}$ expected at energies $\gtrsim 7 \mathrm{keV}$ (e.g. Kallman et al. 2004). The most likely explanation is that the centroid of the line is blueshifted, implying that the material responsible for the observed feature is outflowing. Prime candidates for the origin of the line are the inner K-shell

\footnotetext{
4 http://physics.nist.gov/PhysRefData/ASD/lines_form. html
} 
Table 2. Summary of the Swift (XRT+BAT) parameters of the best-fit model described in Sect. 3, where a high-energy absorption feature is superimposed to a continuum composed by an absorbed power law plus a reflection component, with the addition of a scattered power law.

\begin{tabular}{|c|c|c|c|c|c|c|c|c|c|c|c|}
\hline \multicolumn{5}{|c|}{ Direct, reflected, and scattered continuum } & \multicolumn{4}{|c|}{ Absorption line } & \multirow[b]{2}{*}{$\begin{array}{c}\log F_{2-10 \mathrm{keV}} \\
(10)\end{array}$} & \multirow[b]{2}{*}{$\begin{array}{c}\log L_{2-10 \mathrm{keV}} \\
(11) \\
\end{array}$} & \multirow[b]{2}{*}{$\begin{array}{c}\chi^{2} / \text { d.o.f. } \\
(12)\end{array}$} \\
\hline $\begin{array}{l}N_{\mathrm{H}} \\
(1) \\
\end{array}$ & $\begin{array}{c}\Gamma \\
(2) \\
\end{array}$ & $\begin{array}{l}A_{\mathrm{pl}} \\
(3)\end{array}$ & $\begin{array}{c}R \\
(4) \\
\end{array}$ & $\begin{array}{c}\% \text { scatt. } \\
(5)\end{array}$ & $\begin{array}{c}E \\
(6) \\
\end{array}$ & $\begin{array}{l}\sigma_{\mathrm{E}} \\
(7) \\
\end{array}$ & $\begin{array}{l}A_{\mathrm{E}} \\
(8) \\
\end{array}$ & $\begin{array}{l}E W \\
(9) \\
\end{array}$ & & & \\
\hline $2.4 \pm 0.3$ & $1.8 \pm 0.1$ & $1.8_{-0.3}^{+0.4} \times 10^{-3}$ & $1.4 \pm 0.4$ & $2.1 \pm 0.8$ & $7.8 \pm 0.3$ & $<270$ & $-\left(1.3_{-0.9}^{+1.3}\right) \times 10^{-5}$ & $-\left(230_{-170}^{+390}\right)$ & $-11.19 \pm 0.22$ & $42.61 \pm 0.18$ & $241.5 / 228$ \\
\hline
\end{tabular}

Notes. Errors are quoted at the $90 \%$ confidence level for one parameter of interest $\left(\Delta \chi^{2}=2.71\right)$. Column $(1)$ : intrinsic column density, in units of $10^{22} \mathrm{~cm}^{-2}$. Column (2): power laws (absorbed and unabsorbed) and reflection component photon index. Column (3): absorbed power-law normalization, in units of photons $\mathrm{keV}^{-1} \mathrm{~cm}^{-2} \mathrm{~s}^{-1}$. Column (4): reflection fraction. Column (5): percentage of scattering fraction, defined as the ratio of the unabsorbed and absorbed power-law normalizations. Column (6): rest-frame energy centroid of the Gaussian absorption line, in units of $\mathrm{keV}$. Column (7): absorption line width, in units of eV. Column (8): Gaussian absorption line normalization, in units of photons $\mathrm{cm}^{-2} \mathrm{~s}^{-1}$. Column (9): absorption line equivalent width, in units of eV. Column (10): observed flux (de-absorbed by our Galaxy) in the 2-10 keV energy band, in units of erg cm $\mathrm{cm}^{-2} \mathrm{~s}^{-1}$. Column (11): intrinsic luminosity in the $2-10 \mathrm{keV}$ energy band, in units of erg s $\mathrm{s}^{-1}$. Column (12): $\chi^{2}$ and number of degrees of freedom.

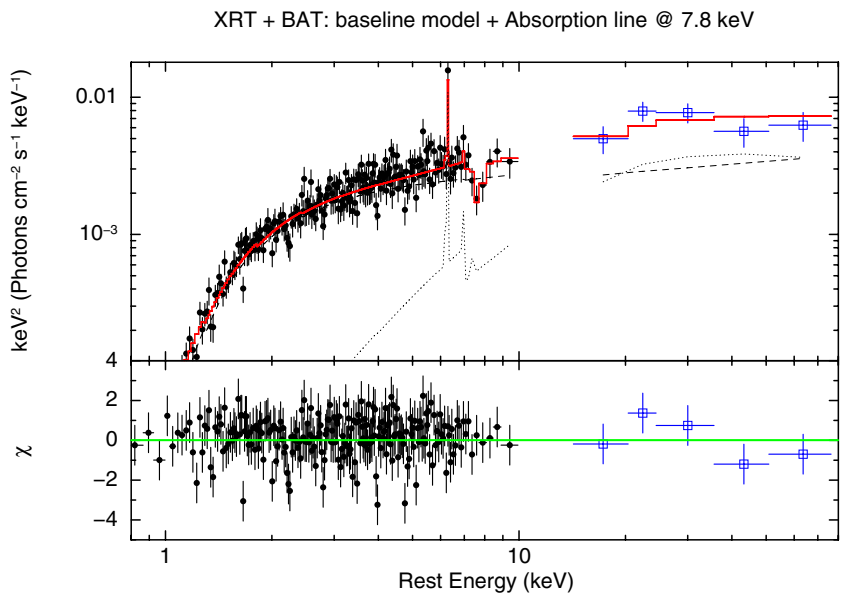

Fig. 3. Upper panel: unfolded XRT data (in black) and average BAT spectrum (in blue) fitted including a Gaussian absorption line $(E \sim$ $7.8 \mathrm{keV}, E W \sim-230 \mathrm{eV}$ and $\sigma_{\mathrm{E}} \sim 190 \mathrm{eV}$ ). The continuum (red continuous line) is modelled with an absorbed plus an unabsorbed power law and a reflection component. Lower panel: relevant residuals, plotted in terms of sigma with error bars of size one.

resonances from moderately highly ionised $\mathrm{Fe} \mathrm{K} \alpha$, as observed in other AGNs. Indeed, assuming $\mathrm{K} \beta$ absorption by moderately ionised $\mathrm{Fe}$, we would also expect to observe strong $\mathrm{K} \alpha$ absorption by the same species (see e.g. the detailed discussion in Markowitz et al. 2006). Being conservative, we can identify the line with the Fe XXVI at $E=6.966 \mathrm{keV}$ resonant absorption; in this case, the observed centroid would indicate a substantial blueshifted velocity of $(0.11 \pm 0.03) c$. An origin in $\mathrm{Fe} \mathrm{K} \alpha$ at a lower ionisation would imply an even higher blueshift.

The observed outflow velocity of $v_{\text {out }} \sim 0.11 c$ would translate into a lower limit on the radial distance, corresponding to the escape radius at which the material is able to leave the system. When a spherical geometry is assumed, this limit is $R \geq$ $R_{\text {esc }}=2 G M / v_{\text {out }}^{2}$, where $M$ is the enclosed mass producing the inward gravitational force. Under the reasonable hypothesis that this corresponds to the mass of the central $\mathrm{BH}$, assuming the estimate reported in the literature $\left(M_{\mathrm{BH}} \sim 1.15 \times 10^{8} M_{\odot}\right.$, see Sect. 2), we have $R_{\text {esc }} \sim 2.7 \times 10^{15} \mathrm{~cm}$. This translates to a possible launch radius of $10^{-3} \mathrm{pc}$, or $\sim 80 r_{\mathrm{S}}$ (Schwarzschild radii, $r_{\mathrm{S}}=2 G M_{\mathrm{BH}} / c^{2}$ ).

The quality of the available data prevents a more detailed spectral analysis, but the ionisation level of the Fe responsible of the absorption and the observed $E W \sim-230 \mathrm{eV}$ (though with large uncertainties) would suggest that we are dealing with a high-ionisation (ionisation parameter ${ }^{5} \log \xi \sim 3 \mathrm{erg} \mathrm{cm} \mathrm{s}^{-1}$ ), high-column density $\left(N_{\mathrm{H}} \sim 10^{23} \mathrm{~cm}^{-2}\right)$ outflow (e.g. Gofford et al. 2013).

\section{Summary}

In this paper, we have reported on our $0.3-10 \mathrm{keV}$ observation of the Seyfert 1.9 galaxy 1SXPS J050819.8+172149, a member of the local LIRG pair known as CGCG 468-002. The Swift-XRT data have been analysed jointly with the Swift-BAT spectrum, averaged over 70 months. The continuum is well described by an intrinsic power law with photon index $\Gamma \sim 1.8$, absorbed by a column of $N_{\mathrm{H}} \sim 2.4 \times 10^{22} \mathrm{~cm}^{-2}$. A reflected component is also observed, with a reflection fraction of $\sim 1.4$, while a weak soft scattered component ( $\sim 2 \%$ of scattering fraction) can account for the observed soft emission. The de-absorbed luminosity is $L_{2-10 \mathrm{keV}} \sim 4 \times 10^{42} \mathrm{erg} \mathrm{s}^{-1}$.

Our Swift monitoring (the first observation of this source extending up to energies $E \sim 7-10 \mathrm{keV}$ ), performed in less than one month, provides us with a tentative detection $(\sim 2.1 \sigma$ significance) of an absorption trough at a rest-frame energy of $\sim 7.8 \mathrm{keV}$. When the feature is described by a simple Gaussian absorption line, its properties (e.g. energy and EW) are consistent with an origin in a material moving with a velocity of $\sim 0.11 c$. To our knowledge, this would be the first detection with Swift of a previously unknown high-velocity outflow.

The low statistics of the data and the high energy of the observed residuals, near the higher boundary of the bandpass of XRT, do not allow us to test more physically consistent models (e.g. grids of photoionised absorbers generated with the XSTAR photoionisation code; Kallman et al. 2004). However, if the detection is confirmed, the observed EW and the derived velocity suggest physical parameters typical of an extremely powerful outflow, as observed in only a handful of AGNs (e.g. the ultra luminous infrared galaxy/quasar Mrk 231; Feruglio et al. 2015). In this case, the kinetic output could match or exceed the typical fraction of bolometric luminosities required for AGN feedback.

In fact, 1SXPS J050819.8+172149 could resemble Mrk 231: the source studied here is hosted in a star forming merging system and (possibly) shows evidence of a powerful

5 The ionisation parameter is defined as $\xi=L_{\text {ion }} / n R^{2}$, where $n$ is the hydrogen number density of the gas (in $\mathrm{cm}^{-3}$ ) and $R$ is the radial distance of the absorbing/emitting material from the central source of $\mathrm{X}$-ray (in cm), while the ionising luminosity $L_{\text {ion }}$ is in units of $\mathrm{erg} \mathrm{s}^{-1}$. 
disk wind. However, these characteristics are combined in 1SXPS J050819.8+172149 with a lower level of activity, both of accretion and star formation, than observed in Mrk 231. This would make 1SXPS J050819.8+172149 exceptional among the extremely powerful AGN winds studied so far. Merging systems like the one hosting this source are the objects where we expect to better observe the interplay between star formation and accretion, since both phenomena can be triggered by galaxy interactions. Indeed, they are the objects where the coexistence of disk winds and molecular outflows has been found so far (e.g. Mrk 231, Feruglio et al. 2015; IRAS F11119+3257, Tombesi et al. 2015). In addition, comparing the accretion and star forming properties reported in Sect. 2, 1SXPS J050819.8+172149 seems to be one of the few examples of a source that, after a recent episode of star formation, is in a transition phase between the star forming-dominated (HII-LIRG) and the accretiondominated (Seyfert-LIRG) state (Alonso-Herrero et al. 2013). The quenching of the star formation can be related (at least partly) to the increase in the AGN activity, as expected from the co-evolution models. An outflow powerful enough to affect the environment beyond the SMBH's gravitational sphere of influence, as the one possibly detected in the XRT data, could in principle play a significant role in this process.

Better statistics and higher resolution observations, extending at energies above $\sim 10 \mathrm{keV}$, are needed in order to confirm the presence of the feature, improve the significance of this detection, and asses the properties of the associated wind, namely the column density and the ionisation state, and then the radial location with respect to the central source. The knowledge of these parameters would allow us to estimate the mass outflow rate and the kinetic power, to be compared with the energetics of the accretion.

Acknowledgements. We are grateful to the referee for the constructive comments that improved the paper. We warmly thank Alessandro Caccianiga for useful discussions. We also want to thank Neil Gehrels, Boris Sbarufatti and the Swift Mission Operation Center for making every effort to get our observations scheduled. This work made use of data supplied by the UK Swift Science Data Centre at the University of Leicester. This research has made use of NASA's Astrophysics Data System. Support from the Italian Space Agency is acknowledged (contract ASI INAF I/037/12/0). The authors acknowledge financial support from the Italian Ministry of Education, Universities and Research (PRIN2010-2011, grant No. 2010NHBSBE).

\section{References}

Ackermann, M., Ajello, M., Allafort, A., et al. 2012, ApJ, 747, 104

Alonso-Herrero, A., Pereira-Santaella, M., Rieke, G. H., \& Rigopoulou, D. 2012, ApJ, 744, 2

Alonso-Herrero, A., Pereira-Santaella, M., Rieke, G. H., et al. 2013, ApJ, 765, 78

Armus, L., Mazzarella, J. M., Evans, A. S., et al. 2009, PASP, 121, 559

Arnaud, K. A. 1996, in Astronomical Data Analysis Software and Systems V, eds. G. H. Jacoby, \& J. Barnes, ASP Conf. Ser., 101, 17

Baumgartner, W. H., Tueller, J., Markwardt, C. B., et al. 2013, ApJS, 207, 19

Blustin, A. J., Page, M. J., Fuerst, S. V., Branduardi-Raymont, G., \& Ashton, C. E. 2005, A\&A, 431, 111

Borguet, B. C. J., Arav, N., Edmonds, D., Chamberlain, C., \& Benn, C. 2013, ApJ, 762, 49
Burrows, D. N., Hill, J. E., Nousek, J. A., et al. 2005, Space Sci. Rev., 120, 165

Cano-Díaz, M., Maiolino, R., Marconi, A., et al. 2012, A\&A, 537, L8

Cicone, C., Maiolino, R., Sturm, E., et al. 2014, A\&A, 562, A21

Condon, J. J., Cotton, W. D., Greisen, E. W., et al. 1998, AJ, 115, 1693

Corral, A., Della Ceca, R., Caccianiga, A., et al. 2011, A\&A, 530, A42

Crenshaw, D. M., \& Kraemer, S. B. 2005, ApJ, 625, 680

Crenshaw, D. M., \& Kraemer, S. B. 2012, ApJ, 753, 75

Crenshaw, D. M., Kraemer, S. B., \& George, I. M. 2003, ARA\&A, 41, 117

Cresci, G., Mainieri, V., Brusa, M., et al. 2015, ApJ, 799, 82

Dai, X., Shankar, F., \& Sivakoff, G. R. 2008, ApJ, 672, 108

Díaz-Santos, T., Charmandaris, V., Armus, L., et al. 2010, ApJ, 723, 993

Díaz-Santos, T., Charmandaris, V., Armus, L., et al. 2011, ApJ, 741, 32

Díaz-Santos, T., Armus, L., Charmandaris, V., et al. 2013, ApJ, 774, 68

Di Matteo, T., Springel, V., \& Hernquist, L. 2005, Nature, 433, 604

Evans, P. A., Beardmore, A. P., Page, K. L., et al. 2007, A\&A, 469, 379

Evans, P. A., Beardmore, A. P., Page, K. L., et al. 2009, MNRAS, 397, 1177

Evans, P. A., Osborne, J. P., Beardmore, A. P., et al. 2014, ApJS, 210, 8

Fabian, A. C. 2012, ARA\&A, 50, 455

Ferrarese, L., \& Merritt, D. 2000, ApJ, 539, L9

Feruglio, C., Maiolino, R., Piconcelli, E., et al. 2010, A\&A, 518, L155

Feruglio, C., Fiore, F., Carniani, S., et al. 2015, A\&A, in press, DOI: $10.1051 / 0004-6361 / 201526020$

Gebhardt, K., Bender, R., Bower, G., et al. 2000, ApJ, 539, L13

Gehrels, N., Chincarini, G., Giommi, P., et al. 2004, ApJ, 611, 1005

Gofford, J., Reeves, J. N., Tombesi, F., et al. 2013, MNRAS, 430, 60

Hopkins, P. F., \& Elvis, M. 2010, MNRAS, 401, 7

Howell, J. H., Armus, L., Mazzarella, J. M., et al. 2010, ApJ, 715, 572

Huchra, J. P., Macri, L. M., Masters, K. L., et al. 2012, ApJS, 199, 26

Inami, H., Armus, L., Charmandaris, V., et al. 2013, ApJ, 777, 156

Kalberla, P. M. W., Burton, W. B., Hartmann, D., et al. 2005, A\&A, 440, 775

Kallman, T. R., Palmeri, P., Bautista, M. A., Mendoza, C., \& Krolik, J. H. 2004, ApJS, 155, 675

King, A., \& Pounds, K. 2015, ARA\&A, in press [arXiv: 1503.05206]

Kollatschny, W., Kotulla, R., Pietsch, W., Bischoff, K., \& Zetzl, M. 2008, A\&A, 484, 897

Kormendy, J., \& Ho, L. C. 2013, ARA\&A, 51, 511

Markowitz, A., Reeves, J. N., \& Braito, V. 2006, ApJ, 646, 783

Mas-Hesse, J. M., Otí-Floranes, H., \& Cerviño, M. 2008, A\&A, 483, 71

Mateos, S., Carrera, F. J., Page, M. J., et al. 2010, A\&A, 510, A35

Matt, G., Fiore, F., Perola, G. C., et al. 1996, MNRAS, 281, L69

Matt, G., Fabian, A. C., Guainazzi, M., et al. 2000, MNRAS, 318, 173

Mineo, S., Gilfanov, M., \& Sunyaev, R. 2012, MNRAS, 419, 2095

Moretti, A., Campana, S., Mineo, T., et al. 2005, in UV, X-Ray, and GammaRay Space Instrumentation for Astronomy XIV, ed. O. H. W. Siegmund, SPIE Conf. Ser., 5898, 360

Morganti, R., Tadhunter, C. N., \& Oosterloo, T. A. 2005, A\&A, 444, L9

Motch, C., Guillout, P., Haberl, F., et al. 1998, A\&AS, 132, 341

Nandra, K., O’Neill, P. M., George, I. M., \& Reeves, J. N. 2007, MNRAS, 382, 194

Petric, A. O., Armus, L., Howell, J., et al. 2011, ApJ, 730, 28

Piconcelli, E., Jimenez-Bailón, E., Guainazzi, M., et al. 2005, A\&A, 432, 15

Porquet, D., Reeves, J. N., Uttley, P., \& Turner, T. J. 2004, A\&A, 427, 101

Ranalli, P., Comastri, A., \& Setti, G. 2003, A\&A, 399, 39

Reynolds, C. S., Fabian, A. C., Makishima, K., Fukazawa, Y., \& Tamura, T. 1994, MNRAS, 268, L55

Sanders, D. B., \& Mirabel, I. F. 1996, ARA\&A, 34, 749

Stierwalt, S., Armus, L., Surace, J. A., et al. 2013, ApJS, 206, 1

Stierwalt, S., Armus, L., Charmandaris, V., et al. 2014, ApJ, 790, 124

Teng, S. H., Veilleux, S., \& Baker, A. J. 2013, ApJ, 765, 95

Tombesi, F., Sambruna, R. M., Reeves, J. N., et al. 2010, ApJ, 719, 700

Tombesi, F., Meléndez, M., Veilleux, S., et al. 2015, Nature, 519, 436

Valiante, E., Lutz, D., Sturm, E., Genzel, R., \& Chapin, E. L. 2009, ApJ, 701, 1814

Véron-Cetty, M.-P., \& Véron, P. 2001, A\&A, 374, 92

Wilms, J., Allen, A., \& McCray, R. 2000, ApJ, 542, 914 